\title{
O papel da inflexibilidade psicológica na relação entre o stress relacionado com a infertilidade e os sintomas depressivos em mulheres com um diagnóstico de infertilidade
}

\author{
The role of Infertility-related psychological inflexibility in the relationship between \\ infertility-related stress and depressive symptoms in women with infertility
}

Beatriz Cardoso (1)

Ana Galhardo $(1,2)$

Ilda Massano-Cardoso

Marina Cunha $(1,2)$

(1) Instituto Superior Miguel Torga, Coimbra, Portugal

(2) Universidade de Coimbra, Centro de Investigação em Neuropsicologia e Intervenção Cognitiva e Comportamental (CINEICC), Portugal

(3) Universidade de Coimbra, Faculdade de Medicina, Portugal

(4) Universidade de Coimbra, Faculdade de Economia, Centro de Estudos e Investigação em Saúde da Universidade de Coimbra (CEISUC), Portugal

Recebido: 11/10/2021; Revisto: 08/11/2021; Aceite: 15/11/2021.

\begin{abstract}
Resumo
Introdução: A infertilidade é uma condição médica e social com impacto na vida das pessoas, nomeadamente a nível conjugal, familiar, social e financeiro. Desta forma, vários estudos apontam para uma comorbilidade entre a psicopatologia e a infertilidade, sendo que quem passa por este processo pode utilizar formas de regulação emocional desadaptativas, traduzidas em inflexibilidade psicológica. Objetivos: O presente estudo pretendeu explorar o papel mediador da inflexibilidade psicológica relacionada com a infertilidade, na relação entre o stress relacionado com a infertilidade e os sintomas depressivos, em mulheres com um diagnóstico de infertilidade a realizar tratamento médico. De acrescentar como objetivo secundário, a exploração de associações entre os anos de diagnóstico e as variáveis em estudo. Metodologia: Conduziu-se um estudo transversal numa amostra de 96 mulheres recrutadas com o apoio da Associação Portuguesa de Fertilidade. As participantes preencheram um conjunto de instrumentos de autorresposta online, tendo sido recolhida informação sociodemográfica e clínica, bem como relativa à avaliação dos níveis de sintomas depressivos, stress relacionado com a infertilidade e inflexibilidade psicológica relacionada com a infertilidade. Resultados: Os resultados obtidos demonstraram que o efeito total da variável stress relacionado com a infertilidade sobre os sintomas depressivos foi de 0,46 , sendo totalmente mediado pela inflexibilidade psicológica relacionada com a infertilidade. Discussão: A dificuldade no alcance de uma gravidez é um acontecimento de vida doloroso e que interfere nos objetivos e planos de construção de uma família, que pode originar stress e sintomas depressivos, sendo a relação entre estes sintomas influenciada pelo mecanismo de inflexibilidade psicológica. Neste sentido, intervenções como a Terapia da Aceitação e Compromisso e o Programa Baseado no Mindfulness para a Infertilidade, ao integrarem como alvo a inflexibilidade psicológica, podem ser particularmente indicadas para esta população.
\end{abstract}

Palavras-Chave: Infertilidade; Inflexibilidade psicológica; Sintomas depressivos; Stress; Estudo transversal descritivo.

\section{DI\&D | ISMT}

rpics@ismt.pt

https://rpics.ismt.pt
Publicação em Acesso Aberto

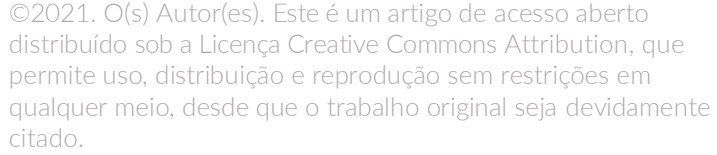

Ana Galhardo

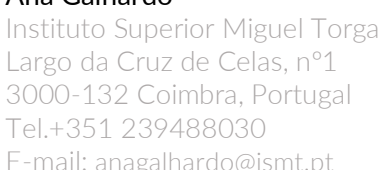




\begin{abstract}
Background and Aim: Introduction: Infertility is a medical and social condition that impacts people's lives at the marital, family, social and financial levels. Several studies point to comorbidity between psychopathology and infertility, and people facing the demands of infertility may use maladaptive mechanisms of emotion regulation translated into psychological inflexibility. Objectives: This current study aimed to explore the mediating role of infertility-related psychological inflexibility in the relationship between infertility-related stress and depressive symptoms in women presenting an infertility diagnosis and pursuing infertility medical treatment. In addition, as a secondary aim, the associations between the time since diagnosis and the study variables were examined. Methods: A cross-sectional study was conducted on a sample of 96 women recruited with the support of the Portuguese Fertility Association. Participants filled in online a set of self-report instruments. Sociodemographic and clinical data were collected, and standardized measures of infertility-related stress, depressive symptoms, and infertility-related psychological inflexibility were used. Results: The results demonstrated that the effect of infertility-related stress on depressive symptoms was .46, being totally mediated by infertility-related psychological inflexibility. Discussion: Difficulty in achieving a pregnancy is a painful life event that interferes with the goals and plans for building a family, which can lead to stress and depressive symptoms. The relationship between these symptoms seems to be influenced by the mechanism of infertility-related psychological inflexibility. Therefore, interventions such as Acceptance and Commitment Therapy and the MindfulnessBased Program for Infertility may be particularly suitable for this population by integrating psychological inflexibility as a therapeutic target.
\end{abstract}

Keywords: Depressive Symptoms; Infertility; Psychological inflexibility; Stress; Descriptive cross-sectional study.

\title{
Introdução
}

A infertilidade é uma doença crónica, caracterizada pela inexistência de uma gravidez, após 12 meses de relações sexuais regulares e desprotegidas (Shapiro, 1988; Zegers-Hochschild et al., 2017). Esta condição médica tem uma prevalência mundial de aproximadamente 9\% (Boivin et al., 2007), sendo que Datta et al. (2016) referem que uma em cada oito mulheres, e um em dez homens apresentam problemas de fertilidade. Em Portugal, segundo um estudo que foi realizado por Silva-Carvalho e Santos (2009), a prevalência encontra-se nos mesmos valores, cerca de 9 a $10 \%$.

O problema da infertilidade pode surgir de fatores femininos, masculinos, desconhecidos ou mistos (quando ambos os membros do casal apresentam problemas de fertilidade) (Lindsay \& Vitrikas, 2015). De referir também que a infertilidade pode ser diferenciada segundo dois tipos, a primária, caracterizada pela incapacidade de o casal alcançar, nem nunca ter conseguido alcançar uma gravidez, e a secundária, quando o casal já conseguiu ter uma gravidez previamente, mas que, no entanto, numa segunda ou mais tentativas de engravidar, estas não são bem-sucedidas (Moura-Ramos, 2011).

A infertilidade é um acontecimento inesperado na vida das pessoas, sendo um obstáculo à prossecução de um objetivo frequentemente tido como fundamental na vida das pessoas, que é a parentalidade (Moura-Ramos, 2011), pois uma parte importante do desenvolvimento e identidade na adultez, está relacionado com a capacidade de reprodução (Cousineau \& Domar, 2007). Desta maneira, o ter de lidar com o diagnóstico de infertilidade e as exigências dos próprios tratamentos, induzem dificuldades psicológicas, podendo variar de reações emocionais negativas transitórias a formas mais graves de sintomatologia psicopatológica (Galhardo et al., 2020). De acordo com Verhaak et al. (2010), cerca de $30 \%$ das pessoas com infertilidade correm o risco de desenvolver sintomas de depressão, ansiedade e 
stress (Szkodziak et al., 2020) clinicamente significativos. Em particular, a depressão (patologia) e o stress (resposta fisiológica e comportamental), caracterizam-se por poderem ter um impacto negativo na fertilidade (feminina e masculina) pois modificam o funcionamento das glândulas endócrinas e do sistema imunológico, podendo resultar numa fertilidade reduzida (Szkodziak et al., 2020).

O stress é considerado um estado de tensão mental ou emocional devido a circunstâncias de vida adversas ou exigentes (Newbegin, 2014), sendo uma experiência subjetiva decorrente da interpretação que a pessoa faz de um acontecimento ameaçador para si (Pozza et al., 2019), como é o caso da infertilidade e dos seus tratamentos. Para além do stress vivenciado aquando do diagnóstico desta doença, é também frequente a existência de sentimentos como choque, tristeza, raiva, frustração, perda da autoestima e perda da autoconfiança (Klock, 2015). O stress relacionado com a infertilidade pode ser um constructo complexo, incluindo diversos domínios relacionados com a infertilidade. Segundo Newton et al. (1999), é possível identificar cinco domínios do stress relacionado com a infertilidade:

1) preocupações sociais (pensamentos sobre a infertilidade, sensibilidade perante comentários de outras pessoas, ou sentimentos de isolamento social, alienação da família e amigos);

2) preocupações sexuais (dificuldade em lidar com a programação das relações sexuais, ou a diminuição da satisfação com a sexualidade);

3) preocupações com a relação (preocupações com o impacto da infertilidade na relação conjugal, dificuldades em falar sobre o problema de fertilidade, e compreender e/ou aceitar as diferenças);

4) desejo da parentalidade (importância da parentalidade como objetivo pessoal essencial à vida);

5) rejeição de um estilo de vida sem filhos (perspetiva negativa de um estilo de vida sem filhos).

Num estudo de Awtani et al. (2017) foi avaliado os níveis de stress em casais a realizar tratamento de inseminação intrauterina (IIU) e fertilização in vitro (FIV), tendo sido a prevalência do stress global elevada, no entanto, considerando os domínios relacionados com a infertilidade de Newton et al. (1999), o domínio do stress relacionado com o desejo da parentalidade foi o mais elevado dos cinco. Tendo sido possível avaliar, que o stress relacionado com a infertilidade é mais elevado nas mulheres comparativamente com os homens, verificando-se o mesmo nos domínios do desejo da parentalidade, preocupações sociais e sexuais. Relativamente ao tipo de tratamento (IIU e FIV) não houve diferenças significativas no que respeita ao stress relacionado com a infertilidade (Awtani et al., 2017).

Apesar do impacto da infertilidade poder ser transitório, não deixa de se revelar num grande sofrimento psicológico, particularmente no que diz respeito à depressão. Com efeito, Fieldsend (2018) menciona que são muitas vezes relatados sentimentos de perda e tristeza referentes à não-maternidade, sendo que o desejo de ter filhos e a consciencialização de não os poder ter, sustentam esta tristeza/angústia. Fieldsend e Smith (2020), numa análise fenomenológica interpretativa, refere que a perda de esperança em ter filhos, é como um luto, em que as pessoas vivem com emoções complexas após tentarem ter sucesso em alcançar uma gravidez o que se relaciona com patologias psicológicas, nomeadamente, com a depressão.

A depressão tem vindo a ser apontada como um problema comum e significativo na infertilidade, quer aquando do diagnóstico, quer durante e após os tratamentos. Vários estudos têm sugerido diferentes valores de prevalência da depressão em mulheres e homens com infertilidade. Por exemplo, Volgsten (2008) refere que $30 \%$ das mulheres e $10 \%$ dos homens, a realizar tratamentos de FIV preenchem 
critérios para a depressão. Por sua vez, Holley et al. (2015) encontraram uma percentagem de 39,1\% de mulheres e $15,3 \%$ de homens que preenchiam os critérios para perturbação depressiva major. Mais recentemente, Joelsson et al. (2017) reportaram uma percentagem de 57,6 \% de mulheres com infertilidade também a apresentar sintomas de depressão e ansiedade. Deka e Sarma (2010) propõem alguns mecanismos pelos quais a depressão afeta diretamente a infertilidade envolvendo a fisiologia do estado depressivo, sendo eles, níveis elevados de prolactina (hormona segregada pela hipófise), interrupção do eixo hipotálamo-pituitária-adrenal e disfunção tireoidiana. Ainda que estes aspetos careçam de um maior conhecimento, sabe-se que em pessoas com infertilidade os sintomas depressivos graves parecem estar significativamente associados a sofrimento pessoal, conjugal e social (Peterson et al., 2014). Em Portugal, num estudo de Galhardo et al. (2011), foi percetível pontuações mais elevadas de depressão em casais com infertilidade que estavam a realizar tratamento médico $(M=8,52, D P=$ $7,40)$, comparadas com as de casais presumivelmente férteis $(M=5,04, D P=4,88)$ e casais com problemas de infertilidade que eram candidatos a adoção como forma de alcançar a parentalidade $(M=$ $4,25, D P=5,92$ ), sendo estas diferenças estatisticamente significativas. A depressão é, desta forma, um preditor de descontinuação do tratamento médico, pois mulheres clinicamente deprimidas não reúnem as condições emocionais necessárias para muitas vezes continuar com o tratamento (Domar et al., 1992; Pedro et al., 2016), sendo também um fator preditor a duração da infertilidade. Domar et al. (1992) referem que a depressão atinge o seu auge durante o terceiro ano, decrescendo posteriormente, dado que durante os dois-três primeiros anos estas pessoas têm esperança e são otimistas em relação aos resultados do tratamento, o que se esbate nos anos seguintes, optando futuramente por outras alternativas, como é o caso da adoção ou da escolha de uma vida sem filhos. Também Lawson et al. (2014) encontraram um padrão semelhante com um aumento da sintomatologia depressiva em pessoas com infertilidade ao longo do tempo de tratamento.

A infertilidade apresenta consequências a nível psicológico, manifestando-se de diversas formas, entre as quais sintomatologia depressiva e de stress. Contudo, torna-se relevante explorar o papel de mecanismos, por exemplo de regulação emocional, que possam estar relacionados com o emergir deste tipo de sintomas. Noutras populações, o mecanismo de inflexibilidade psicológica tem amplamente sido associado a sintomatologia psicopatológica (e.g., Chawla \& Ostafin, 2007; Lilly \& Allen, 2015), mas este tem sido menos abordado em estudos com população com infertilidade. A inflexibilidade psicológica, de acordo com a concetualização da Terapia da Aceitação e Compromisso (ACT), é o núcleo do sofrimento psicológico. A inflexibilidade psicológica é caracterizada como um mecanismo transdiagnóstico, traduzido em esforços rígidos para controlar reações psicológicas a coisas desagradáveis, indesejadas ou experiências privadas dolorosas, como, por exemplo, pensamentos, emoções, memórias, sensações corporais e predisposições comportamentais (Harris, 2009; Hayes et al., 1999; Levin et al., 2014). Este mecanismo inclui uma indisponibilidade para estar em contacto com experiências internas, que são aversivas ou desagradáveis, ou ações para modificar essas experiências (Hayes \& Smith, 2005). A inflexibilidade psicológica corresponde, assim, à tendência para evitar, controlar ou minimizar a experiência de eventos privados stressantes, e para agir em discordância com os seus valores. Esta inflexibilidade psicológica, a curto prazo, pode oferecer alívio e revelar-se uma estratégia de regulação emocional (Hayes et al., 1996; Kashdan et al., 2006; Tavakoli et al., 2019). No entanto, quando se torna 
num padrão rígido e inflexível, acaba por se tornar num processo mal adaptativo, estando associada a psicopatologia e a uma diminuição da qualidade de vida (Chawla \& Ostafin, 2007; Lilly \& Allen, 2015). Vários estudos indicam que a inflexibilidade psicológica está relacionada com outros constructos da saúde mental (e.g., depressão, ansiedade, qualidade de vida, autoeficácia); como um preditor, mediador ou moderador, numa variedade de condições de saúde, como a dor crónica, doença inflamatória intestinal, cancro da mama e problemas relacionados com o peso (Costa \& Pinto-Gouveia, 2011; Ferreira et al., 2016; González-Fernández et al., 2017; Lilly \& Allen, 2015). Especificamente na área da infertilidade, Galhardo et al. (2019) encontraram um efeito indireto do impacto da infertilidade em diversas áreas da vida, em que sintomas depressivos eram mediados pelo evitamento experiencial (um dos componentes da inflexibilidade psicológica), sugerindo assim que este constructo deve ser considerado nas avaliações e intervenções em pessoas com infertilidade. Assim, no contexto da infertilidade, a inflexibilidade psicológica pode traduzir-se na indisponibilidade para estar em contacto com acontecimentos privados aversivos (e.g., pensamentos dolorosos acerca das suas dificuldades em alcançar a parentalidade, respostas emocionais como a tristeza ou o stress) e agir de forma discordante com os seus valores, por exemplo evitando reuniões familiares ou com amigos nas quais estejam presentes crianças, quando na realidade o estar presente para a família e amigos seria um dos seus valores. Os indivíduos podem também evitar assuntos relacionados com estes pensamentos/ sentimentos, procurando suprimi-los (Galhardo et al., 2020).

Atendendo a que especificamente na área da infertilidade, o contributo da inflexibilidade psicológica no sofrimento das pessoas afetadas por esta condição médica e social se encontra pouco examinado, o objetivo principal deste estudo, é o de explorar o papel mediador da inflexibilidade psicológica relacionada com a infertilidade, na relação entre o stress relacionado com a infertilidade e os sintomas depressivos, em mulheres com um diagnóstico de infertilidade a realizar tratamento médico para esta condição. Hipotetiza-se que as preocupações sociais, sexuais, relativas à relação conjugal, assim como o desejo de parentalidade e a rejeição de um estilo de vida sem filhos, que integram o stress relacionado com a infertilidade, possam ter um efeito direto sobre os sintomas depressivos experienciados por estas mulheres, mas que esse efeito possa igualmente ser mediado pela inflexibilidade psicológica (indisponibilidade para estar em contacto com estes sintomas de stress e esforços para os suprimir ou modificar) (Modelo 1). Além disso, atendendo a que a relação entre o stress relacionado com a infertilidade e a sintomatologia depressiva pode acontecer no sentido inverso, isto é, os sintomas depressivos terem um efeito direto sobre o stress, podendo esse efeito ser também mediado pela inflexibilidade psicológica relacionada com a infertilidade, pretendeu-se testar o modelo alternativo (Modelo 2).

Um outro objetivo secundário foi o de explorar em que medida a variável clínica anos de diagnóstico se associava com os sintomas depressivos, stress relacionado com a infertilidade e com a inflexibilidade psicológica relacionada com a infertilidade. Esta exploração assenta na hipótese de que uma maior duração da infertilidade pode acarretar maiores níveis de sintomatologia depressiva e de stress. Por outro lado, o padrão inverso é igualmente plausível, atendendo a que com o passar do tempo as mulheres podem desenvolver estratégias adaptativas para lidar com a situação de infertilidade, traduzindo-se em menor sintomatologia depressiva e de stress. 


\section{Método}

\section{Participantes}

A amostra foi composta por 96 mulheres portuguesas com um diagnóstico de infertilidade autorreportado. As características sociodemográficas e clínicas da mesma são reportadas na Tabela 1.

\section{Tabela 1}

\section{Caracterização Sociodemográfica e Clínica da Amostra}

\begin{tabular}{lcc}
\hline Variáveis & $M$ & $D P$ \\
\hline Idade & 36,62 & 4,29 \\
Escolaridade & 15,60 & 3,24 \\
Anos de Diagnóstico & 4,85 & 3,88 \\
\hline & $n$ & $\%$ \\
\hline Estado Civil & 56 & 58,3 \\
Casadas & 40 & 41,7 \\
União de Facto & & \\
Causas de Infertilidade & 42 & 43,8 \\
Fator feminino & 20 & 20,8 \\
Fator masculino & 17 & 17,7 \\
Fator misto & 17 & 17,7 \\
Etiologia desconhecida & & \\
Tratamentos anteriores & 75 & 78,1 \\
Já haviam realizado tratamento médico & 21,9 \\
A realizar pela primeira vez & 21 & \\
Tipo de tratamento atual & & 36,5 \\
Aguardavam marcação do tratamento & 35 & 30,2 \\
Aguardavam o resultado de testes/exames & 29 & 12,5 \\
A realizar FIV & 12 & 12,5 \\
A realizar ICSI & 12 & 5,2 \\
A receber estimulação hormonal (não sendo seguida de FIV ou ICSI) & 5 & 3,1 \\
A realizar IIU & 3 & \\
\hline
\end{tabular}

Nota. $N=96$. FIV= Fertilização in vitro; ICSI = injeção intracitoplasmática de espermatozoide; IIU = inseminação intrauterina.

A média das idades das participantes rondou os 36 anos, tendo variado entre 27 a 44 anos. A maioria era casada e, em média apresentava uma escolaridade ao nível do ensino superior.

Relativamente às variáveis clínicas, as participantes foram diagnosticadas com infertilidade há aproximadamente cinco anos. Quanto às causas da infertilidade, o fator feminino foi o mais representado, seguido do masculino, feminino e masculino combinados e, por último, de fator desconhecido. A maioria 
já havia realizado tratamento para a infertilidade. No que se refere à fase de tratamento em que se encontravam, as categorias a aguardar pela marcação do tratamento ou por resultados de testes ou exames foram as mais indicadas.

\section{Instrumentos}

No presente estudo utilizou-se um questionário sociodemográfico que possibilitou a recolha de dados relativos à idade, anos de escolaridade e estado civil. No que se refere a variáveis clínicas relacionadas com a infertilidade foi utilizado um questionário que integrou perguntas relativas ao tempo decorrido após o diagnóstico, etiologia, realização de tratamentos médicos anteriores e tratamento a ser realizado aquando da recolha dos dados. De referir que a informação acerca das variáveis clínicas foi autorreportada, não tendo existido consulta de processos médicos.

\section{Fertility Problem Inventory (FPI)}

O Inventário de Problemas de Fertilidade (Newton et al., 1999; versão portuguesa de Moura-Ramos et al., 2012), é um instrumento de autorresposta de 46 itens, que avalia o stress relacionado com a infertilidade. Os participantes são questionados sobre até que ponto concordam ou discordam de preocupações ou crenças, utilizando a escala que varia de Discordo totalmente (0) a Concordo totalmente (6). O FPI permite avaliar cinco subescalas: "preocupação social", "preocupação sexual", "preocupação com a relação", "desejo da parentalidade" e "rejeição de estilo de vida sem filhos". O FPI permite avaliar cinco subescalas: 1) preocupação social (e.g., "As reuniões de família são especialmente difíceis para mim"), 2) preocupação sexual (e.g., "Considero ter deixado de ter prazer na relação sexual devido ao problema de fertilidade"), 3) preocupação com a relação (e.g., "Aborrece-me que o/a meu/minha companheiro/a reaja de forma diferente ao problema"), 4) desejo de parentalidade (e.g., "É difícil sentir-me como um verdadeiro adulto até ter um(a) filho/a") e 5) rejeição de um estilo de vida sem filhos (e.g., "Não ter um(a) filho/a (ou outro/a) permitir-me-ia ter tempo para fazer outras coisas gratificantes"). Pontuações mais altas são indicadoras de níveis mais elevados de stress relacionado com a infertilidade. O FPI e as suas subescalas revelaram uma boa consistência interna, com o score total a apresentar um valor de alfa de Cronbach de 0,93 (Newton et al., 1999). O valor do alfa de Cronbach no presente estudo foi de 0,92.

\section{Depression, Anxiety and Stress Scales - 21 (DASS-21)}

A Escala de Ansiedade, Depressão e Stress (Lovibond \& Lovibond, 1995; versão portuguesa de PaisRibeiro et al., 2004) é um instrumento de 21 itens que mede os sintomas de depressão (e.g., "Senti que não tinha muito valor como pessoa"), de ansiedade (e.g., "Senti-me quase a entrar em pânico") e de stress (e.g., "Dei por mim a ficar agitado"). Os itens são respondidos numa escala variando de Não se aplicou nada a mim (0) a Aplicou-se a mim a maior parte das vezes (3), relatando a frequência dos sintomas durante a semana anterior. Pontuações mais elevadas nas subescalas da DASS-21 traduzem níveis mais elevados de sintomas emocionais negativos. A DASS-21 revela boas propriedades psicométricas. As subescalas de depressão, ansiedade e stress na versão original (Lovibond \& Lovibond, 1995) revelam um valor do alfa de Cronbach de 0,94, 0,87 e 0,91, enquanto que, na versão portuguesa (Pais-Ribeiro et al., 2004) foi de 0,84, 0,80 e 0,87 , respetivamente. No atual estudo, apenas foi utilizada a escala relativa aos sintomas depressivos, apresentando esta um valor de alfa de Cronbach de 0,91. 


\section{Psychological Inflexibility Scale - Infertility (PIS-I)}

A Escala de Inflexibilidade Psicológica relacionada com a Infertilidade (Galhardo et al., 2020) foi desenvolvida com o objetivo de avaliar a inflexibilidade psicológica em pessoas que enfrentam problemas de fertilidade. É constituída por um conjunto de 16 itens (e.g., "Sinto que sou uma pessoa incompleta devido a este problema de fertilidade") em que são respondidos numa escala a variar entre Nunca verdadeiro (1) a Sempre verdadeiro (7). Pontuações mais altas na PIS-I revelam um maior nível de inflexibilidade psicológica relacionada com a infertilidade. A PIS-I apresenta uma excelente consistência interna com um alfa de Cronbach de 0,95 (Galhardo et al., 2020). No presente estudo o alfa de Cronbach encontrado foi de 0,93.

\section{Procedimentos Metodológicos}

No que concerne ao desenho, este estudo foi do tipo analítico observacional e de desenho transversal. O estudo foi alvo de aprovação pela Direção da Associação Portuguesa de Fertilidade (associação de pacientes). As participantes foram convidadas a participar no estudo através de um post publicado nas redes sociais e no website da Associação Portuguesa de Fertilidade. A participação envolveu o preenchimento online dos instrumentos de autorresposta descritos anteriormente. Todas as participantes tiveram acesso a informação acerca dos objetivos e âmbito do estudo, sendo salvaguardados o anonimato e a confidencialidade dos dados recolhidos. A participação era de caráter voluntário, sendo obrigatório o fornecimento do consentimento informado antes do preenchimento do protocolo de avaliação e os dados recolhidos foram tratados de forma agregada. Todos os procedimentos seguidos apresentavam concordância com os princípios éticos inerentes à investigação com seres humanos, estando em conformidade com a Declaração de Helsínquia de 1964 e as suas reformulações posteriores. De referir ainda ter sido facultado às participantes um contacto de email do estudo para clarificação de dúvidas ou solicitação de informações adicionais.

\section{Procedimentos Analíticos}

Os dados foram analisados por recurso ao software estatístico Statistical Package for the Social Sciences (SPSS, v.27) e ao AMOS (v.26). para efeitos de caracterização da amostra relativamente às variáveis sociodemográficas e clínicas recorreu-se a estatística descritiva com cálculo de médias e desvios padrão para as variáveis contínuas e de frequências e percentagens para as variáveis categoriais. A comparação dos valores médios das variáveis com valores médios de referência dessas mesmas variáveis foi efetuada através do cálculo de testes $t$ de Student para uma amostra. Para a análise da associação entre as variáveis foram calculados os coeficientes de correlação de Pearson. De acordo com Dancey e Reidy (2017), coeficientes com valores entre 0,10 e 0,39 são considerados fracos, entre 0,40 e 0,69 moderados e acima de 0,70 fortes. As análises de trajetórias (path analysis) foram levadas a cabo através de análise de equações estruturais com método da máxima verosimilhança. Para avaliar o ajustamento do modelo de mediação testado foram usados os seguintes indicadores: o teste do $\chi^{2}$, o Comparative Fit Index (CFI), o Goodness of Fit Index (GFI), o Tucker and Lewis Index (TLI), e o Root Mean Square Error of Approximation (RMSEA). De acordo com Marôco (2010), valores mais baixos no teste do $\chi^{2}$, e $p>0,05$ revelam um bom ajustamento e valores de CFI, GFI e TLI entre 0,90 e 0,95 são indicadores de bom ajustamento e valores 
superiores ou iguais a 0,95 de um ajustamento muito bom. Relativamente ao RMSEA e igualmente de acordo com as recomendações de Marôco (2010), valores 0,05 e 0,10 traduzem um bom ajustamento e valores inferiores ou iguais a 0,05, um muito bom ajustamento.

\section{Resultados}

Previamente à condução da path analysis, foram examinadas as estatísticas descritivas das variáveis do modelo hipotetizado (Tabela 2). Os valores de skewness e kurtosis de todas as variáveis encontravam-se dentro dos valores recomendados por Hair et al. (2010), pelo que foi considerada a inexistência de violações ao pressuposto da normalidade.

\section{Tabela 2}

Descritivas e Correlações entre as Variáveis em Estudo

\begin{tabular}{lcccc}
\hline Medidas & $M$ & $D P$ & 1 & 2 \\
\hline 1. FPI & 158,58 & 35,06 & - & \\
2. EADS-Depressão & 7,82 & 5,53 & $0,52^{* * *}$ & - \\
3. PIS-I & 65,43 & 22,87 & $0,72^{* * *}$ & $0,64^{* * *}$
\end{tabular}

Nota. $N=96$. FPI = Fertility Problem Inventory; EADS-Depressão = Subescala de depressão das Escalas de Ansiedade, Depressão e Stress; PIS-I = Psychological Inflexibility Scale - Infertility.

${ }^{* * *} p<0,001$.

Os valores médios do stress relacionado com a infertilidade foram comparados com os reportados no estudo de validação da versão portuguesa do FPI (Moura-Ramos et al., 2012). Os resultados mostraram a existência de diferenças estatisticamente significativas $t_{(95)}=7,50 ; p<0,001 ; d=0,77$, com as participantes do presente estudo a reportarem valores mais altos. Quanto aos valores médios dos sintomas depressivos (subescala de depressão da EADS-21), a sua comparação com os valores indicados num estudo anterior com jovens adultos e adultos portugueses (Pinto et al., 2015) foi reveladora da existência de diferenças estatisticamente significativas $\left(t_{(95)}=6,82 ; p<0,001 ; d=0,47\right)$, com as mulheres da presente amostra a apresentarem valores mais elevados. No que se refere à comparação do score médio na PIS-I da presente amostra comparativamente com o score encontrado no estudo de desenvolvimento desta medida (Galhardo et al., 2020), não foram observadas diferenças estatisticamente significativas.

De seguida foram calculadas as correlações, não se observando a existência de multicolinearidade, dado que todos os valores foram inferiores a 0,90 (Hair et al., 2010). Os resultados das correlações entre as variáveis em estudo são apresentados na Tabela 2.

O modelo hipotetizado foi testado através de um modelo saturado (Figura 1), com seis parâmetros. Todas as trajetórias demonstraram ser estatisticamente significativas, à exceção do caminho do stress relacionado com a infertilidade para os sintomas depressivos $\left(b=0,022 ; S E_{\mathrm{b}}=0,018 ; Z=1.240 ; p=0,215\right.$; $\beta=0,140$ ). Como tal, esta trajetória foi removida e o modelo recalculado. 


\section{Figura 1}

Modelo Teórico de Mediação da Inflexibilidade Psicológica Relacionada com a Infertilidade na Relação entre o Stress Relacionado com a Infertilidade e os Sintomas Depressivos

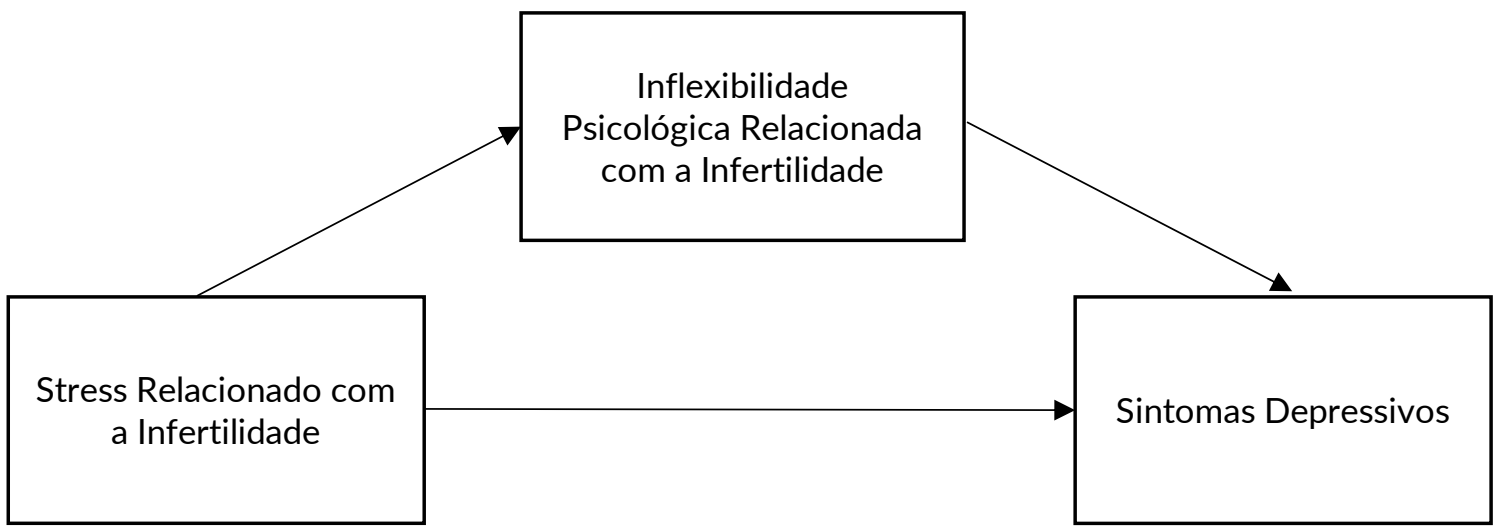

No segundo modelo ajustado (Figura 2), todas as trajetórias se revelaram significativas, explicando 40\% da variância, e o modelo revelou um bom ajustamento $\left(\chi^{2}=1,527 ; g I=1 ; p=0,901 ; C F I=0,995 ; G F I=\right.$ 0,989; $T L I=0,986 ;$ RMSEA =0,074 e MECVI = 0,126). De referir ter-se verificado uma diminuição do MECVI de 0,132 no primeiro modelo testado para 0,126, o que é indicador de que o modelo apresenta um ajustamento superior ao anteriormente testado (Marôco, 2010). Observou-se que o efeito total da variável stress relacionado com a infertilidade sobre os sintomas depressivos foi de 0,46 , sendo totalmente mediado pela inflexibilidade psicológica relacionada com a infertilidade.

\section{Figura 2}

Modelo de Mediação Total da Inflexibilidade Psicológica Relacionada com a Infertilidade na Relação entre o Stress Relacionado com a Infertilidade e os Sintomas Depressivos

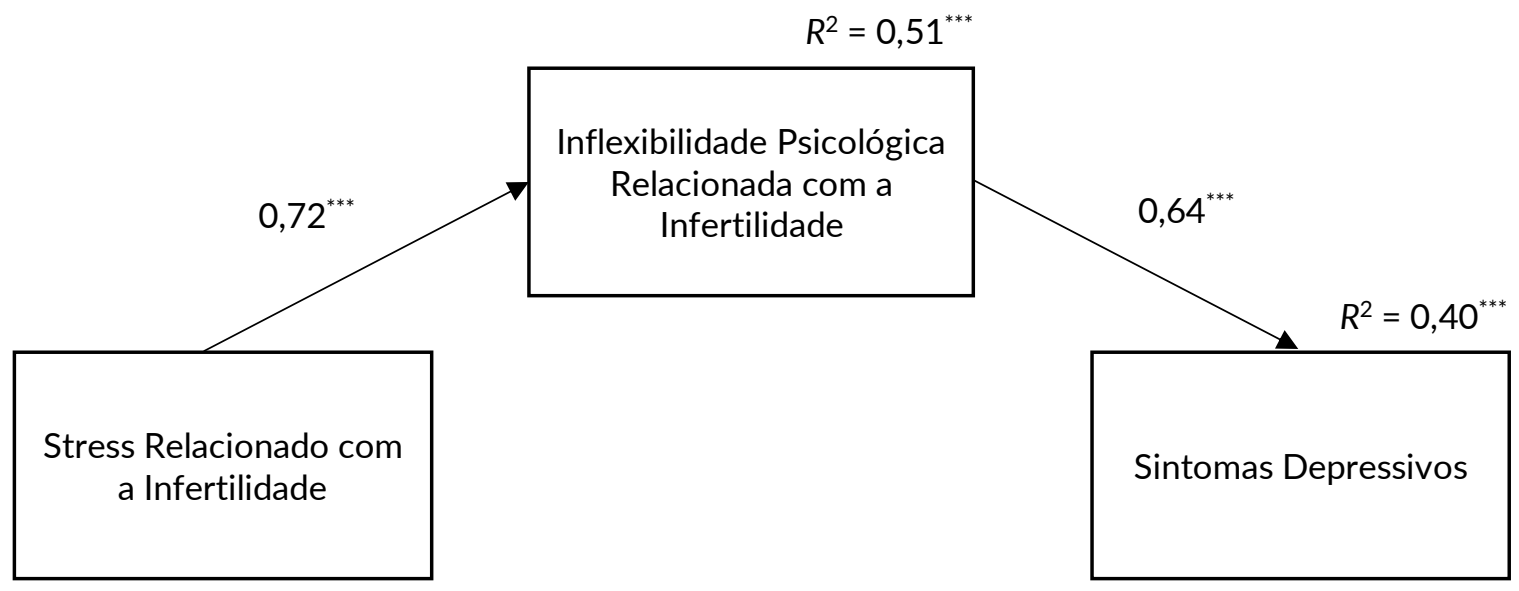

Nota. São apresentados os valores estandardizados dos coeficientes das trajetórias entre as variáveis em estudo. ${ }^{* * *} p<0,001$. 
O modelo alternativo (Modelo 2), no qual os sintomas depressivos poderão ter um efeito direto sobre o stress relacionado com a infertilidade, mas também um efeito de mediação da inflexibilidade psicológica relacionada com a infertilidade, com seis parâmetros, foi igualmente testado. Todas as trajetórias demonstraram ser estatisticamente significativas, à exceção do caminho dos sintomas depressivos para o stress relacionado com a infertilidade $\left(b=0,72 ; S E_{\mathrm{b}}=0,58 ; Z=1,24 ; p=0,215 ; \beta=0,64\right)$. No Modelo 2 reespecificado, após eliminação desta trajetória, todas as trajetórias se mostraram significativas. Este modelo explicou $51 \%$ da variância e revelou um bom ajustamento $\left(\chi^{2}=1,527 ; g I=1 ; p=0,217 ; C F I=\right.$ 0,995; GFI = 0,989; $T L I=0,986 ;$ RMSEA = 0,074; $M E C V I=0,126$ ).

Relativamente à associação entre os anos de diagnóstico e os sintomas depressivos, não se verificou a existência de uma correlação estatisticamente significativa ( $r=0,06 ; p=0,558$ ); observando-se o mesmo padrão em relação ao stress relacionado com a infertilidade $(r=-0,09 ; p=0,360)$.

\section{Discussão}

O presente trabalho teve por objetivo explorar o papel mediador da inflexibilidade psicológica relacionada com a infertilidade, na relação entre o stress relacionado com a infertilidade e os sintomas depressivos, em mulheres com um diagnóstico de infertilidade a realizar tratamento médico. Não foi observada a existência de um efeito direto do stress relacionado com a infertilidade sobre os sintomas depressivos, o que é indicador do papel relevante que a forma como as mulheres lidam com as preocupações sociais, sexuais, com a relação conjugal, o seu desejo de maternidade e a rejeição de um estilo de vida sem filhos desempenha no desenvolvimento de sintomatologia depressiva. Na realidade, foi possível observar que o efeito do stress relacionado com a infertilidade sobre os sintomas depressivos foi totalmente mediado pela inflexibilidade psicológica relacionada com a infertilidade, o que demonstra ser congruente com outros estudos (Galhardo et al., 2019; Kashdan et al., 2006; Levin et al., 2014). Segundo os autores supracitados, o recurso à inflexibilidade psicológica tende a atenuar transitoriamente o sofrimento decorrente da infertilidade, no entanto, intensifica os sintomas depressivos a longo prazo.

A inflexibilidade psicológica, por sua vez, engloba duas componentes, a resistência em permanecer em contacto com experiências internas aversivas (sensações corporais, emoções, pensamentos, memórias e predisposições comportamentais) e a inatividade da ação para mudar experiências aversivas e/ou eventos que as desencadeiam (Hayes \& Smith, 2005). Por exemplo, a experiência de ter de lidar com o diagnóstico de infertilidade e as exigências do tratamento médico pode levar a um aumento de pensamentos negativos da pessoa em relação a si mesma, e a sintomas depressivos e de ansiedade (Galhardo et al., 2019), contribuindo para o evitamento de situações como encontros com amigos ou família onde possa haver crianças, ou o evitamento destes pensamentos, suprimindo-os. Desta forma, situações de stress relacionado com a infertilidade traduzidas em preocupações sociais, conjugais e sexuais, e o desejo da parentalidade ou a rejeição de um estilo de vida sem filhos, estão associadas ao aumento de sintomas depressivos (Newton et al., 1999). Assim, a importância da maternidade na vida de alguém e a rejeição de uma vida sem filhos, pode contribuir para o aumento destas preocupações, levando a estados emocionais difíceis. Durante estes estados emocionais, a utilização do evitamento experiencial como forma de não 
estar em contacto com experiências aversivas pode contribuir para o aparecimento/agravamento de sintomas depressivos (Chawla \& Ostafin, 2007).

Ao ser testado o modelo alternativo (Modelo 2), no qual se hipotetizava que os sintomas depressivos poderiam ter um efeito direto sobre o stress, podendo esse efeito ser também mediado pela inflexibilidade psicológica relacionada com a infertilidade, observaram-se resultados idênticos ao anteriormente testado (Modelo 1). Ainda que estes resultados remetam para a existência de circularidade entre estas variáveis, do ponto de vista clínico parece-nos mais plausível o Modelo 1. É de realçar que pensamentos associados com a importância da maternidade na vida da mulher (identificação com o papel de mãe, perceção da maternidade como um objetivo de vida central) e com a rejeição de um estilo de vida sem filhos (visão negativa deste estilo de vida, consideração de que a felicidade está dependente da maternidade), bem como preocupações sociais (sensibilidade a comentários dos outros, sentimentos de isolamento social), preocupações sexuais (diminuição do prazer sexual, relações sexuais planeadas), e preocupações com a relação conjugal (diferenças na forma como lidam com a infertilidade) poderão estar mais facilmente associados a sintomas depressivos do que o contrário.

De acordo com a literatura, tem-se observado um reconhecimento crescente, no que diz respeito ao melhoramento da saúde mental, e mais especificamente a sintomas depressivos entre mulheres com infertilidade, de abordagens terapêuticas que ajudam na regulação emocional, diminuindo a inflexibilidade psicológica, entre as quais a Terapia da Aceitação e Compromisso (ATC) (Peterson \& Eifert., 2011) ou o Programa Baseado no Mindfulness para a Infertilidade (PBMI) (Galhardo et al., 2013). Este último revelou ser um programa eficaz de intervenção psicológica, desenvolvido especificamente para mulheres que experienciam infertilidade, contribuindo particularmente para a diminuição dos sintomas depressivos, e para o aumento de competências de mindfulness e de uma maior perceção de autoeficácia para lidar com a infertilidade (Galhardo et al., 2013). Os sintomas depressivos estão frequentemente relacionados com pensamentos autorreferenciais distorcidos (e.g., inutilidade e de autoculpabilização), sendo que a descentração em relação a esses pensamentos e a observação dos mesmos, é uma das competências treinadas durante o PBMI, sendo um fator crucial para a diminuição de variáveis psicopatológicas, como é o caso dos sintomas depressivos (Galhardo et al., 2013, 2018). A depressão está também relacionada com o entrapment e a derrota, sendo que no estudo de Galhardo et al. (2013) parece ter havido uma redução nestas variáveis por meio deste programa, uma vez que as mulheres percebem e compreendem melhor o mundo exterior, assim como as suas experiências, existindo uma menor motivação para a fuga (evitamento experiencial). Através da prática de mindfulness, a experiência interna e externa torna-se mais consciente, havendo um foco no momento presente, existindo uma atitude de abertura e curiosidade, não sendo necessária uma modificação ou supressão em relação a pensamentos dolorosos ou sentimentos relacionados com o passado ou futuro (Galhardo et al., 2013). Há, por assim dizer, maior capacidade de aceitação das experiências internas (Bond, 2011; Hayes \& Smith, 2005), que tornam estas mulheres mais capazes de tomarem decisões de forma deliberada e consciente, guiadas por valores, de forma mais flexível (Harris, 2009).

Efetivamente, o diagnóstico de infertilidade é difícil para os indivíduos, o casal (quando as pessoas se encontram numa relação), e para toda a família, causando mudanças significativas nos planos e objetivos que têm para a sua vida, podendo originar sentimentos de derrota, falta de realização no papel de mãe/pai, 
mas também dificuldades conjugais, causando desconforto físico, emocional e financeiro. Os nossos resultados, no que respeita à inexistência de associação entre os anos de diagnóstico com as variáveis em estudo, vão ao encontro do reportado por Domar et al. (1992) e Lawson et al. (2014). De facto, é de referir que as mulheres que participaram neste estudo apresentavam uma média de anos de diagnóstico de infertilidade próxima dos cinco anos, o que pode ajudar a explicar a inexistência da referida associação, nomeadamente com os sintomas depressivos. Ainda que não tenham sido exploradas diferenças entre grupos com distintas durações do diagnóstico de infertilidade em virtude do tamanho da presente amostra, tal deveria ser explorado em estudos futuros.

No que diz respeito às limitações do estudo, é de referir que, apesar de o tamanho da amostra ser adequado para a condução das análises, esta apenas englobava participantes do sexo feminino, pelo que em estudos futuros a inclusão de pessoas do sexo masculino poderá ser relevante, dado que, apesar de ser na mulher que esta condição médica tem mais impacto, o companheiro pode também revelar problemas a nível psicológico ao lidar com este diagnóstico. A participação (online) tem também algumas limitações, como a sub-representação da população, o que restringe a possibilidade de se fazer generalizações (Wright, 2005). Desta forma, investigação futura nesta área poderá ser feita numa amostra mais alargada e incluindo participantes do sexo masculino, com o objetivo de se perceber em que medida a inflexibilidade psicológica relacionada com a infertilidade atua de forma idêntica à encontrada em mulheres.

Apesar das limitações referidas, o presente estudo apontou para a existência de uma relação entre o stress relacionado com a infertilidade e os sintomas depressivos, sendo esta relação totalmente mediada pela inflexibilidade psicológica especificamente relacionada com a infertilidade, sugerindo a relevância de avaliar este construto e, no caso de se verificarem níveis elevados do mecanismo de inflexibilidade psicológica, poder recorrer a intervenções terapêuticas dirigidas a uma diminuição da inflexibilidade psicológica, o que por sua vez pode ter impacto nos sintomas depressivos e no stress relacionado com a infertilidade nesta população.

Agradecimentos | Acknowledgements: Os autores gostariam de agradecer à Associação Portuguesa de Fertilidade pelo apoio na divulgação do estudo e a todas as participantes pela colaboração.

Conflito de interesses | Conflict of interest: nenhum | none.

Fontes de financiamento | Funding sources: nenhuma | none.

Contributos: BC: Pesquisa bibliográfica; Revisão da literatura; Análise estatística; Escrita do manuscrito; AG: Desenho do estudo; Preparação das medidas e escrita do protocolo; Análise estatística; Supervisão da escrita do manuscrito e aprovação do manuscrito final; IMC: Análise estatística e aprovação do manuscrito final; MC: Desenho do estudo; Revisão e aprovação do manuscrito final.

\section{Referências}

Awtani, M., Mathur, K., Shah, S., \& Banker, M. (2017). Infertility stress in couples undergoing intrauterine insemination and in vitro fertilization treatments. Journal of Human Reproductive Sciences, 10(3), 221-225. https://doi.org/g53b 
Boivin, J., Bunting, L., Collins, A. J., \& Nygren, G. A. (2007). International estimates of infertility prevalence and treatment-seeking: potential need and demand for infertility medical care. Human Reproduction, 22(6), 1506-1512. https://doi.org/c27zhm

Bond, F. W., Hayes, S. C., Baer, R. A., Carpenter, K. M., Guenole, N., Orcutt, H. K., Waltz, T., \& Zettle, R. D. (2011). Preliminary psychometric properties of the Acceptance and Action Questionnaire-II: A revised measure of psychological inflexibility and experiential avoidance. Behavior Therapy, 42(4), 676-688. https://doi.org/ftbs23

Chawla, N., \& Ostafin, B. (2007). Experiential avoidance as a function dimensional approach to psychopathology: An empirical review. Journal of Clinical Psychology, 63(9), 871-890. https://doi.org/d69c2j

Costa, J., \& Pinto-Gouveia, J. (2011). The mediation effect of experiential avoidance between coping and psychopathology in chronic pain. Clinical Psychology and psychotherapy, 18(1), 34-37. https://doi.org/dz6tbx

Cousineau, T. M., \& Domar, A. D. (2007). Psychological impact of infertility. Best Practice \& Research Clinical Obstetrics and Gynaecology, 21(2), 293-308. https://doi.org/bcd664

Dancey, C. P., \& Reidy, J. (2017). Statistics without maths for psychology (7a ed.). Pearson Education Inc.

Datta, J., Palmer, M. J., Tanton, C., Gibson, L. J., Jones, K. G., Macdowall, W., Glasier, A., Sonnenberg, P., Field, N., Mercer, C. H., Johnson, A. M., \& Wellings, K. (2016). Prevalence of infertility and help seeking among 15000 women and men. Human Reproduction, 31(9), 2108-2118. https://doi.org/gjpzcv

Deka, P. K., \& Sarma, S. (2010). Psychological aspects of infertility. British Journal of Medical Practitioners, 3(3), 32-34. https://bit.ly/3wPKZpD

Domar, A. D., Broome, A., Zuttermeister, P. C., Seibel, M., \& Friedman, R. (1992). The prevalence and predictability of depression in infertile women. Fertility and Sterility, 58(6), 1158-1163. https://doi.org/g53r

Ferreira, C., Trindade, I. A., \& Martinho, A. (2016). Explaining rigid dieting in normal-weight women: The key role of body image inflexibility. Eating and Weight Disorders, 21(1), 49-56. https://doi.org/f8tvzb

Fieldsend, M. (2018). What is it like being involuntarily childless? Searching for ways of understanding from a psychological perspective. Em N. Sappleton (Ed.), Voluntary and involuntary childlessness (pp. 49-70). Emerald Publishing Limited. https://doi.org/g53q

Fieldsend, M., \& Smith, J. A. (2020). "Either stay grieving or deal with it": The psychological impact of involuntary childlessness for women living in midlife. Human Reproduction, 35(4), 876-885. https://doi.org/g53p

Galhardo, A., Alves, J., Moura-Ramos, M., \& Cunha, M. (2019). Infertility-related stress and depressive symptoms-the role of experiential avoidance: A cross-sectional study. Journal of Reproductive and Infant Psychology, 38(2), 139-150. https://doi.org/g53n

Galhardo, A., Cunha, M., Monteiro, B., \& Pinto-Gouveia, J. (2020). Assessing psychological inflexibility in infertility: The development and validation study of the Psychological Inflexibility Scale - Infertility (PIS-I). Journal of Contextual Behavioral Science, 18, 239-246. https://doi.org/g53m

Galhardo, A., Cunha, M., \& Pinto-Gouveia, J. (2013). Mindfulness-Based Program for Infertility: Efficacy study. Fertility and Sterility, 100(4), 1059-1067. https://doi.org/f2m74g

Galhardo, A., Moura-Ramos, M., Cunha, M., \& Pinto-Gouveia, J. (2018). How does the Mindfulness-Based Program for Infertility (MBPI) work in reducing depressive symptoms?. Mindfulness, 9, 629-635. https://doi.org/gc98b8

Galhardo, A., Pinto-Gouveia, J., Cunha, M., \& Matos, M. (2011). The impact of shame and self-judgment on psychopathology in infertile patients. Human Reproduction, 26(9), 2408-2414. https://doi.org/dk5fmv

González-Fernández, S., Fernández-Rodríguez, C., Mota-Alonso, M. J., García-Teijido, P., Pedrosa, I., \& PérezÁlvarez, M. (2017). Emotional state and psychological flexibility in breast cancer survivors. European Journal of Oncology Nursing, 30, 75-83. https://doi.org/gckwhf

Hair, J. F., Black, W. C., Babin, B. J., \& Anderson, R. E. (2010). Multivariante data analysis (7a ed.). Pearson Education.

Harris, R. (2009). ACT made simple. New Harbinger Publications. 
Hayes, S. C., \& Smith, S. (2005). Get out of your mind and into your life: The new acceptance and commitment therapy. New Harbinger Publications, Inc.

Hayes, S. C., Strosahl, K. D., \& Wilson, K. G. (1999). Acceptance and commitment therapy: An experiential approach to behavior change. Guilford Press.

Hayes, S. C., Wilson, K. W., Gifford, E. V., Follette, V. M., \& Strosahl, K. (1996). Experiential avoidance and behavioral disorders: A functional dimensional approach to diagnosis and treatment. Journal of Consulting and Clinical Psychology, 64(6), 1152-1168. https://doi.org/cgw8mg

Holley, S. R., Pasch, L. A., Bleil, M. E., Gregorich, S., Katz, P. K., \& Adler, N. E. (2015). Prevalence and predictors of major depressive disorder for fertility treatment patients and their partners. Fertility and Sterility, 103(5), 1332-1339. https://doi.org/f27tk7

Joelsson, L. S., Tydén, T., Wanggren, K., Georgakis, M. K., Stern, J., Berglund, A., \& Skalkidou, A. (2017). Anxiety and depression symptoms among sub-fertile women, women pregnant after infertility treatment, and naturally pregnant women. European Psychiatry, 45, 212-219. https://doi.org/gb2ff6

Kashdan, T. B., Barrios, V., Forsyth, J. P., \& Steger, M. F. (2006). Experiential avoidance as a generalized psychological vulnerability: Comparisons with coping and emotion regulation strategies. Behaviour Research and Therapy, 44(9), 1301-1320. https://doi.org/ftqdk2

Klock, S. C. (2015). Reproductive psychology and fertility counseling. Em S. N. Covington (Ed.) Fertility counseling clinical guide and case studies (pp. 33-45). Cambridge University Press. https://bit.ly/3kMVCEF

Lawson, A. K., Klock, S. C., Pavone, M. E., Hirshfeld-Cytron, J., Smith, K. N., \& Kazer, R. R. (2014). Prospective study of depression and anxiety in female fertility preservation and infertility patients. Fertility and Sterility, 102(5), 1377-1384. https://doi.org/f2vzsc

Levin, M. E., MacLane, C., Daflos, S., Seeley, J. R., Hayes, S.C., Biglan, A., \& Pistorello, J. (2014). Examining psychological inflexibility as a transdiagnostic process across psychological disorders. Journal of Contextual Behavioral Science, 3(3), 155-163. https://doi.org/ggjs6c

Lilly, M. M., \& Allen, C. E. (2015). Psychological inflexibility and psychopathology in 9-1-1 telecommunicators. Journal of Traumatic Stress, 28(3), 262-266. https://doi.org/10.1002/jts.22004

Lindsay, T. J., \& Vitrikas, K. R. (2015). Evaluation and treatment of infertility. American Family Physician, 91(5), 308-314. https://www.aafp.org/afp/2015/0301/p308.html

Lovibond, P. F., \& Lovibond, S. H. (1995). The structure of negative emotional states: Comparison of the Depression Anxiety Stress Scales (DASS) with the Beck Depression and Anxiety Inventories. Behaviour Research and Therapy, 33(3), 335-343. https://doi.org/fwhsp7

Marôco, J. (2010). Análise de equações estruturais: Fundamentos teóricos, software \& aplicações. ReportNumber.

Moura-Ramos, M. C. B. (2011). Adaptação psicossocial de casais portugueses à infertilidade e à reprodução medicamente assistida. [Tese de Doutoramento, Faculdade de Psicologia e Ciências da Educação da Universidade de Coimbra]. Repositório científico da Universidade de Coimbra. http://hdl.handle.net/10316/18478

Moura-Ramos, M., Gameiro, S., Canavarro, M. C., \& Soares, I. (2012). Assessing infertility stress: Re-examining the factor structure of the Fertility Problem Inventory. Human Reproduction, 27(2), 496-505. https://doi.org/bhb6ft

Newbegin, C. (2014). The stress epidemic. InnovAiT, 8(1), 36-40. https://doi.org/g53h

Newton, C. R., Sherrard, W., \& Glavac, I. (1999). The Fertility Problem Inventory: Measuring perceived infertility- related stress. Fertility and Sterility, 72(1), 54-62. https://doi.org/bsbw8j

Pais-Ribeiro, J. L., Honrado, A., \& Leal, I. (2004). Contribuição para o Estudo da Adaptação Portuguesa das Escalas de Ansiedade, Depressão e Stress (EADS) de 21 itens de Lovibond e Lovibond. Psicologia, Saúde \& Doenças, 5(2), 229-239. https://hdl.handle.net/10216/6910 
Pedro, J., Sobral, M. P., Mesquita-Guimarães, J., Leal, C., Costa, M. E., \& Martins, M. V. (2016). Couples' discontinuation of fertility treatments: A longitudinal study on demographic, biomedical, and psychosocial risk factors. Journal of Assisted Reproduction and Genetics, 34(2), 217-224. https://doi.org/f9xf4h

Peterson, B. D., \& Eifert, G. H. (2011). Using acceptance and commitment therapy to treat infertility stress. Cognitive and Behavioral Practice, 18(4), 577-587. https://doi.org/bqnm7h

Peterson, B. D., Sejbaek, C. S., Pirritano, M., \& Schmidt, L. (2014). Are severe depressive symptoms associated with infertility- related distress in individuals and their partners?. Human Reproduction, 29(1), 76-82. https://doi.org/f5jwcs

Pinto, J. C., Martins, P., Pinheiro, T. B., \& Oliveira, A. C. (2015). Ansiedade, depressão e stresse: um estudo com jovens adultos e adultos portugueses. Psicologia, Saúde \& Doenças, 16(2), $148-163$. https://www.redalyc.org/pdf/362/36242128002.pdf

Pozza, A., Dèttore, D., \& Coccia, M. E. (2019). Depression and anxiety in pathways of medically assisted reproduction: The role of infertility stress dimensions. Clinical Practice \& Epidemiology in Mental Health, 15, 101-109. https://doi.org/g53g

Shapiro, S. A. (1988). Psychological consequences of infertility. Em J. Zuckerberg (Ed.), Critical psychophysical passages in the life of a woman (pp. 269-289). Springer. https://doi.org/g53f

Silva-Carvalho, J. L., \& Santos, A. (2009). Estudo Afrodite caracterização da infertilidade em Portugal I- Estudo na comunidade. Universidade do Porto.

Szkodziak, F., Krzyżanowski, J., \& Szkodziak, P. (2020). Psychological aspects of infertility: A systematic review. Journal of International Medical Research, 48(6), 1-13. https://doi.org/g53d

Tavakoli, N., Broyles, A., Reid, E., Sandoval, J. R., \& Correa-Fernández, V. (2019). Psychological inflexibility as it relates to stress, worry, generalized anxiety, and somatization in an ethnically diverse sample of college students. Journal of contextual Behavioral Science, 11, 1-19. https://doi.org/ggk8sr

Verhaak, C. M., Lintsen, A. M. E., Evers, A. W. M., \& Braat, D. D. M. (2010). Who is at risk of emotional problems and how do you know? Screening of women going for IVF treatment. Human Reproduction, 25(5), 12431240. https://doi.org/ddfb9t

Volgsten, H., Svanberg, A. S., Ekselius, L., Lundkvist, Ö., \& Poromaa, I. S. (2008). Prevalence of psychiatric disorders in infertile women and men undergoing in vitro fertilization treatment. Human Reproduction, 23(9), 2056-2063. https://doi.org/bhpc6q

Wright, K. B. (2005). Researching Internet-based populations: Advantages and disadvantages of online survey research, online questionnaire authoring software packages, and web survey services. Journal of ComputerMediated Communication, 10(3). https://doi.org/d7z5cr

Zegers-Hochschild, F., Adamson, G. D., Dyer, S., Racowsky, C., De Mouzon, J., Sokol, R., Rienzi, L., Sunde, A., Schmidt, L., Cooke, I. D., Simpsom, J. L., \& Van Der Poel, S. (2017). The international glossary on infertility and fertility care, 2917. Human Reproduction, 32(9), 1786-1801. https://doi.org/gh7gqp 